\title{
Macropinocytosis of type XVII collagen induced by bullous pemphigoid IgG is regulated via protein kinase $C$
}

\author{
Hiroaki Iwata', Mayumi Kamaguchi ${ }^{2}$, Hideyuki Ujiie ${ }^{1}$, Machiko Nishimura ${ }^{1}$, Kentaro Izumi $^{1}$, Ken Natsuga ${ }^{1}$, \\ Satoru Shinkuma ${ }^{1}$, Wataru Nishie ${ }^{1}$ and Hiroshi Shimizu ${ }^{1}$
}

Macropinocytosis is an endocytic pathway that is involved in the nonselective fluid uptake of extracellular fluid. Bullous pemphigoid (BP) is an autoimmune subepidermal blistering disease associated with autoantibodies to type XVII collagen (COL17), which is a component of hemidesmosome. When keratinocytes are treated with BP-IgG, COL17 internalizes into cells by way of the macropinocytosis. We investigated the mechanism of COL17 macropinocytosis using DJM- 1 cells, a cutaneous squamous cell carcinoma cell line. First, non-hemidesmosomal COL17 was preferentially depleted by stimulation with the BP-lgG in the DJM-1 cells. To investigate the signaling involved in COL17-macropinocytosis, the inhibition of small GTPase family members Rac1 and Cdc42 was found to strongly repress COL17 internalization; in addition, the Rho inhibitor also partially blocked that internalization, suggesting these small GTPases are involved in signaling to mediate COL17-macropinocytosis. Western blotting using Phostag-SDS-PAGE demonstrated high levels of COL17 phosphorylation in DJM-1 cells under steady-state condition. Treatment with BP-IgG increased the intracellular calcium level within a minute, and induced the overabundant phosphorylation of COL17. The overabundant phosphorylation of COL17 was suppressed by a protein kinase C (PKC) inhibitor. In addition, PKC inhibitor repressed COL17 endocytosis using cell culture and organ culture systems. Finally, the depletion of COL17 was not observed in the HEK293 cells transfected COL17 without intracellular domain. These results suggest that COL17 internalization induced by BP-lgG may be mediated by a PKC pathway. In summary, BP-lgG initially binds to COL17 distributed on the plasma membrane, and COL17 may be internalized by means of a macropinocytic pathway related to the phosphorylation of the intracellular domain by PKC.

Laboratory Investigation (2016) 96, 1301-1310; doi:10.1038/labinvest.2016.108; published online 24 October 2016

Bullous pemphigoid (BP) is an autoimmune subepidermal blistering disease that demonstrates characteristic clinical, histological, and immunological features of tense blisters with widespread erythema, blistering along the lamina lucida, and the presence of circulating autoantibodies against hemidesmosomal molecules, respectively. ${ }^{1}$ The autoantibodies include two major antigens: type XVII collagen (COL17, also known as BP180) and BP230. In the structure of hemidesmosomes, the COL17 spans from the lamina lucida to the lamina densa. ${ }^{2}$ COL17 is the most likely pathogenic molecule in $\mathrm{BP}$, and the non-collagenous 16A (NC16A) domain is regarded as the major pathogenic epitope for BP. ${ }^{1,3}$ Although the precise mechanisms of blistering after autoantibodies bind to antigens remain unclear, several blistering mechanisms have been proposed, such as activation of inflammatory cells and complements. ${ }^{4-6}$ Kitajima et $\mathrm{ll}^{7}$ reported that COL17 exists in the lateral-apical plasma membrane in pools that are not bound to keratin filaments in cell culture systems. The stimulation of autoantibodies causes immune complexes of COL17 and BP-IgG to be internalized in keratinocytes. ${ }^{8-10}$ COL17 is depleted in keratinocytes after internalization. ${ }^{11}$ In this process, COL17 internalization is mediated by a macropinocytic pathway. ${ }^{10,12}$

Endocytosis is important in regulating external signals into cells, in coordinating membrane recycling and in mediating signal transduction. ${ }^{13}$ There are several endocytic pathways, and the receptor tyrosine kinases are the most studied of the endocytosis mechanisms. Macropinocytosis is an endocytic pathway that shows nonselective fluid uptake of extracellular fluid and that is involved in antigen presentation in dendritic cells and macrophages or in entry by pathogens. ${ }^{14-16}$ Although the definition of macropinocytosis is still

\footnotetext{
${ }^{1}$ Department of Dermatology, Hokkaido University Graduate School of Medicine, Sapporo, Japan and ${ }^{2}$ Department of Oral Diagnosis and Medicine Hokkaido University, Graduate School of Dental Medicine, Sapporo, Japan

Correspondence: Dr H Iwata, MD, PhD, Department of Dermatology, Hokkaido University Graduate School of Medicine, North 15 West 7, Kita-ku, Sapporo 060-8638, Japan. E-mail: hiroaki.iwata@med.hokudai.ac.jp
}

Received 22 July 2016; revised 21 August 2016; accepted 31 August 2016 
controversial because of the absence of specific markers, it is known to be coordinated with actin cytoskeleton reorganization which is suppressed by amiloride inhibiting membrane ruffling. ${ }^{14,17}$ Macropinosome formation and maturation, which are mediated by actin cytoskeleton reorganization, are controlled by small GTPases and phosphoinositides. ${ }^{18}$ Rho GTPases are members of the small GTPase family and contain three major groups: Rho, Rac and Cdc42. ${ }^{19} \mathrm{Racl}$ is an important signal for macropinocytosis in various cell types, ${ }^{20}$ and $\mathrm{Cdc} 42$ is also reported to be activated in macropinocytosis in some cell types. ${ }^{20,21}$ However, it is unclear whether Rho, Rac and Cdc42 are involved in the macropinocytosis process activated by BP-IgG binding to $\mathrm{COL} 17$ on the keratinocytes. To investigate the precise blistering mechanisms including cellular signaling could lead to find a possible therapeutic target. ${ }^{22}$ Indeed, many signaling molecules, such as PI3K or p38, are already found for the therapeutic targets in autoimmune blistering diseases. ${ }^{22}$

Here we investigate the mechanism of COL17 macropinocytosis generated when BP-IgG binds to COL17. We demonstrate that COL17 internalization via macropinocytosis is mediated by the phosphorylation of the intracellular domain of COL17 through protein kinase C (PKC) and by involvement of Rho, Rac and Cdc42 activations.

\section{MATERIALS AND METHODS BP Patients' IgG and Mouse Monoclonal Anti-human COL17-NC16A IgG}

The patients' serum were pooled from BP patients fulfilling the following inclusion criteria: (i) clinical blistering or erosions on the skin; and (ii) circulating autoantibodies against COL17 as detected by BP180-NC16A ELISA/CLEIA (MBL, Nagoya, Japan). Total IgG was purified using protein $G$ affinity columns according to the manufacturer's instructions (GE Healthcare, Amersham, UK). Mouse monoclonal anti-human COL17-NC16A IgG (TS39-3) was produced previously. ${ }^{23}$ In accordance with the Hokkaido University Hospital bylaws and standard operating procedures approved by the Hokkaido University Hospital Review Board, we obtained patient consent for experimental procedures to be performed at Hokkaido University Hospital from each participating patient upon his or her first visit to the hospital. The studies were conducted according to the Helsinki Guidelines.

\section{Humanized COL17 Mice, and Skin Organ Culture}

The ear skin of a humanized COL17 mouse ${ }^{24}$ was obtained and torn into two pieces. This skin was placed on a polycarbonate membrane filter with the dermal side down (Isopore $0.45 \mu \mathrm{m}$ pore size, Merck Millipore, Darmstadt, Germany), and cultured with DMEM. The epidermis on the polycarbonate filter was placed on the DMEM with PKC inhibitor at $37^{\circ} \mathrm{C}$ for $1 \mathrm{~h}$ followed by culturing with BP-IgG at $37^{\circ} \mathrm{C}$ for $2 \mathrm{~h}$.

\section{Chemical Inhibitors}

We used several inhibitors for macropinocytosis and protein kinase. The following were added into the culture medium $1 \mathrm{~h}$ before IgG stimulation: trihydrochloride as a Racl inhibitor (Sigma Aldrich, St, Luis, MO, $200 \mu \mathrm{M}$ in $\mathrm{H}_{2} \mathrm{O}$ ), dihydrochloride as a Rho-associated coiled-coil forming protein kinase (ROCK) inhibitor (Sigma Aldrich, St Luis, MO, $10 \mu \mathrm{M}$ in $\mathrm{H}_{2} \mathrm{O}$ ), ML141 as a Cdc42 inhibitor (Sigma Aldrich, St Luis, $\mathrm{MO}, 10 \mu \mathrm{M}$ in DMSO), IPA-3 as a p21-activated kinase 1 (PAK1) inhibitor (Sigma Aldrich, St Luis, MO, $25 \mu \mathrm{M}$ in DMSO), and GF109203X as a PKC inhibitor (Sigma Aldrich, St, Luis, MO, $5 \mu \mathrm{M}$ in DMSO).

\section{Cell Cultures}

Normal human epidermal keratinocytes (NHEK) obtained from uninvolved skin of surgical specimens for benign skin tumors were cultured first in progenitor cell-targeted medium containing $0.05 \mathrm{mM}$ calcium (CELLnTEC, Bern, Switzerland). When used for experiments, they were cultured in Dulbecco's modified Eagle's medium (DMEM) (Life Technologies, Tokyo, Japan), containing $1.8 \mathrm{mM}$ calcium for $24 \mathrm{~h}$. An antibiotic and antimycotic solution (Sigma Aldrich, St Luis, $\mathrm{MO}$ ) and $10 \%$ fatal calf serum were supplied in the medium. DJM-1 cells isolated from human skin squamous cell carcinoma ${ }^{25}$ and human COL17-transfected human embryonic kidney (HEK) 293 cells (COL17-293 cells) ${ }^{26}$ were cultured in DMEM containing $1.8 \mathrm{mM}$ calcium. In some experiments, DJM-1 cells were cultured in DMEM with $0.05 \mathrm{mM}$ calcium (Life Technologies, Tokyo, Japan).

The mutated COL17-293 cells (COL17-delta 1-423 cells), lacking the intracellular domain Met 1-Asp 423, were generated using COL17-293 cells (Figure 5a). Briefly, human COL17A1 cDNA in pcDNA5/FRT was digested by EcoRV to delete the intracellular domain (Met 1-Asp 423), and then a synthesized DDDDK-tag was introduced into the digested COL17-pcDNA5/FRT vector. The mutant COL17A1 cDNA in pcDNA5/FRT vector was transfected into Flp-in-293 cells.

To investigate the internalization and depletion of COL17, cells were cultured to $\sim 40 \%$ confluence. ${ }^{11}$ BP-IgG (final concentration $1 \mathrm{mg} / \mathrm{ml}$ ), normal human IgG (final concentration $1 \mathrm{mg} / \mathrm{ml}$ ), TS39-3 (final concentration $2.5 \mu \mathrm{g} / \mathrm{ml}$ ), and PBS were added to the culture medium and incubated.

\section{Quantitative RT-PCR}

To confirm the effects of calcium in the culture medium, KRT1 expression was measured. mRNA was extracted from DJM- 1 cells cultured in high- and low-calcium medium $24 \mathrm{~h}$ after plating by RNeasy Mini Kit (Qiagen, Valencia, CA). Single-stranded cDNA was synthesized using RT2 First Strand Kit (Qiagen, Valencia, CA). According to the manufacturer's instructions, assays were performed using $\mathrm{RT}^{2}$ SYBR GREEN/ ROX PCR Master Mix (Qiagen) and Step-OnePlus (Applied Biosystems). KRT1 gene expressions were analyzed. Relative expression ratios were normalized to glyceraldehydes 3phosphate dehydrogenase (GAPDH). 


\section{Immunofluorescent Microscopy}

For immunofluorescent staining, the cells were cultured on fourwell chamber slides (Thermo Fisher Scientific, Rockford, IL) or type I collagen-coated eight-well chamber slides (Becton, Dickinson and Company, Franklin Lakes, NJ). The cells were washed with PBS and fixed with $4 \%$ paraformaldehyde at room temperature for $15 \mathrm{~min}$. After permeabilization with $0.25 \%$ TritonX-100 for $10 \mathrm{~min}$, the cells were incubated with 100 -fold diluted FITC-conjugated anti-human IgG (Jackson ImmunoResearch Laboratories, West Grove, PA) or anti-mouse IgG (Jackson ImmunoResearch, West Grove, PA). The nuclei were stained with DAPI (Life Technologies, Tokyo, Japan). Photoimages were taken with a Keyence fluorescence microscopy (Keyence, Osaka, Japan). IgG was quantified by ImageJ (http://rsbweb.nih.gov/ij/).

\section{Western Blotting and Phostag Western Blotting}

For western blot analysis of whole-cell lysates, cells were lysed in RIPA buffer (Thermo Fisher Scientific, Rockford, IL) containing a protease inhibitor cocktail (Sigma Aldrich, St Louis, MO) and the lysates were centrifuged. For analysis of TritonX-100-soluble or -insoluble pools, cells were lysed in $1 \%$ TritonX-100 in PBS containing a protease inhibitor cocktail (Sigma Aldrich, St Louis, MO), followed by centrifugation, and the resultant supernatant was stored as the cytosol/membrane fraction. TritonX-100-insoluble pellets were solubilized in 2\% SDS, $62.5 \mathrm{mM}$ Tris- $\mathrm{HCl}, \mathrm{pH} 6.8,30 \%$ glycerol and stored as the cytoskeleton fraction, which includes whole insoluble hemidesmosomal components. For Phostag-western blot analysis of whole-cell lysates, cells were lysed with NP40 buffer (50 mM Tris- $\mathrm{HCl}, \mathrm{pH} 7.4,150 \mathrm{mM}$ $\mathrm{NaCl}, 1 \% \mathrm{NP}-40,0.5 \%$ sodium deoxycholate, $0.1 \%$ SDS) containing a protease inhibitor cocktail (Sigma Aldrich, St, Louis, MO) and a phosphatase inhibitor cocktail (Wako Pure Chemical Industries, Osaka, Japan), and then the lysates were centrifuged. Each fraction was subjected to SDS-PAGE in $6 \%$ polyacrylamide gel. For Phostag Western blotting,
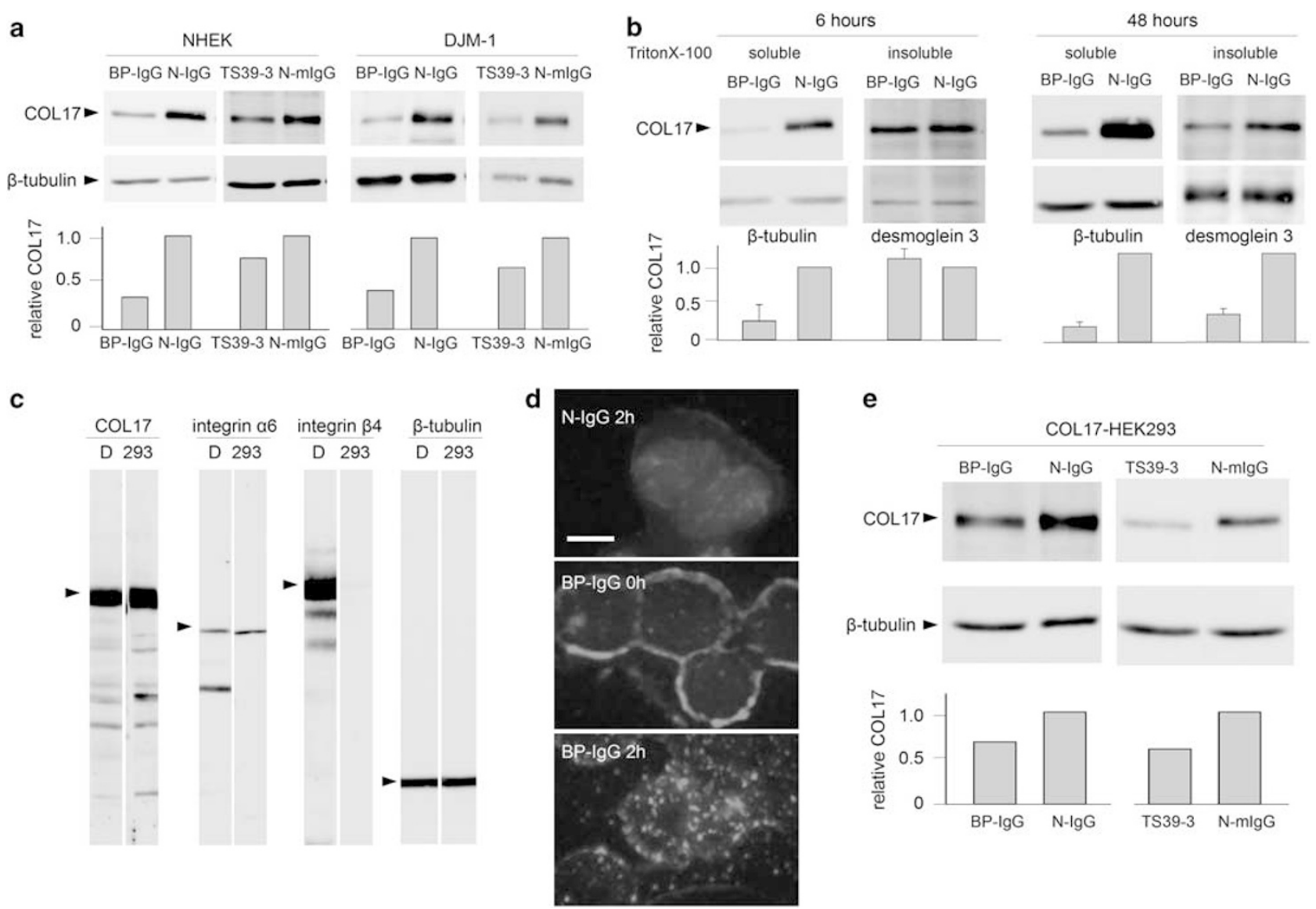

Figure 1 Non-hemidesmosomal COL17 is preferentially internalized and depleted. (a) COL17 from NHEKs (left) and DJM-1 cells (right) was treated with BP-IgG and mouse monoclonal anti-human COL17-NC16A lgG (TS39-3) for $6 \mathrm{~h}$. COL17 was detected by the rabbit anti-C-terminus of human COL17. (b) The cells were fractionated into TritonX-100-soluble (left) and -insoluble (right) pools 6 or $48 \mathrm{~h}$ after BP-lgG treatment. COL17-depletion is detected in the TritonX-100-insoluble fraction 48 h, but not 6 h, after BP-lgG stimulation (right). $\beta$-tubulin and desmoglein 3 are loading controls for TritonX-100 soluble and TritonX-100 insoluble fractions, respectively. (c) COL17 and integrin $a 6$ are expressed equivalently in DJM-1 and human COL17-transfected HEK 293 (COL17-293) cells, but integrin $\beta 4$ is hardly detected in COL17-293 cells. $\beta$-tubulin is a loading control. (d) COL17 internalization induced by BPIgG is observed in COL17-293 cells. (e) COL17-293 cells were treated with BP-IgG or TS39-3 for $24 \mathrm{~h}$. The upper panels are representative western blots; the lower panels are relative COL17 normalized with normal lgG (a, b, and e). N-IgG, normal human IgG; N-mlgG, normal mouse IgG; D, DJM-1 cell; 293, COL17-293 cell. Scale bar, $10 \mu \mathrm{m}$. 
a
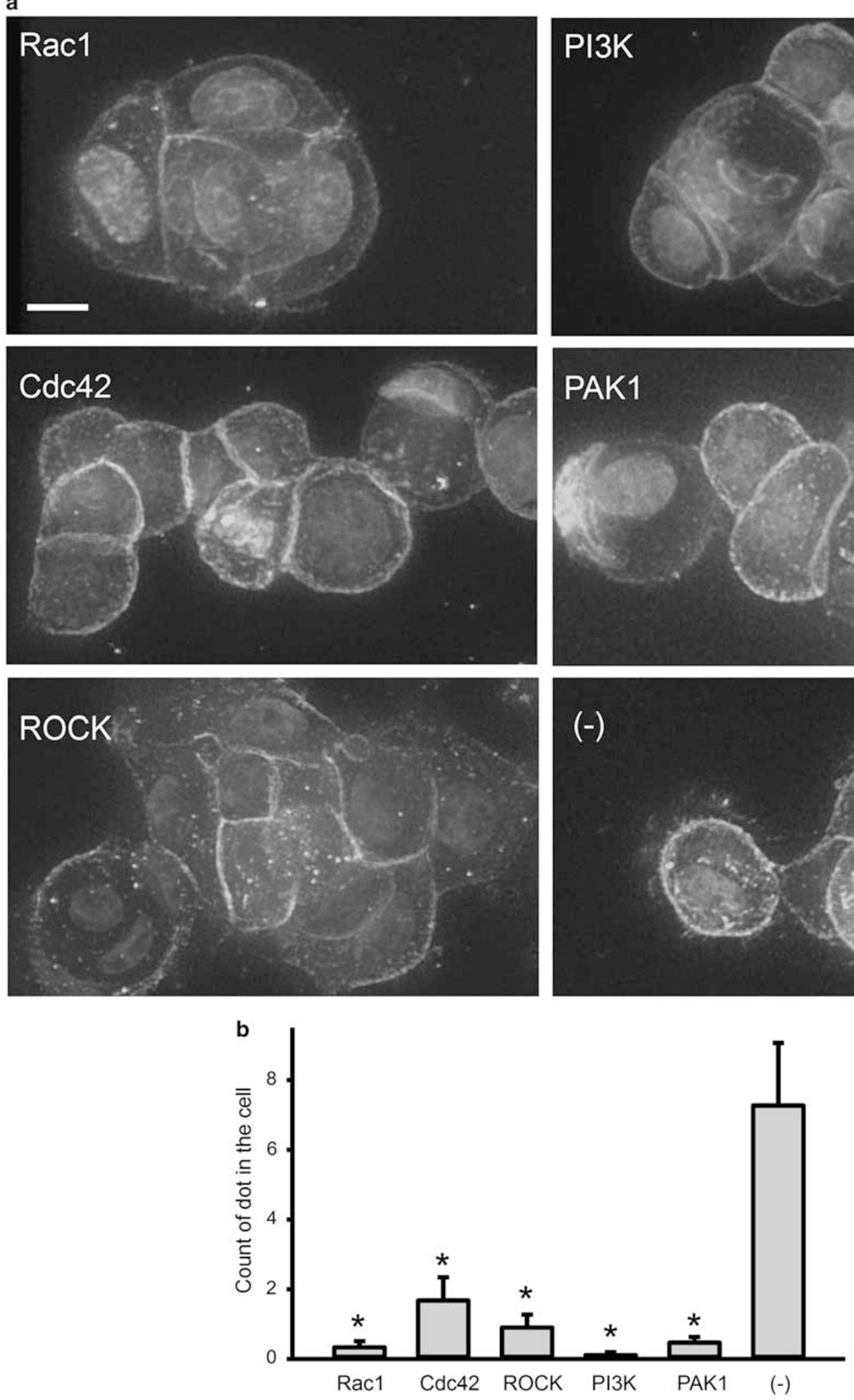
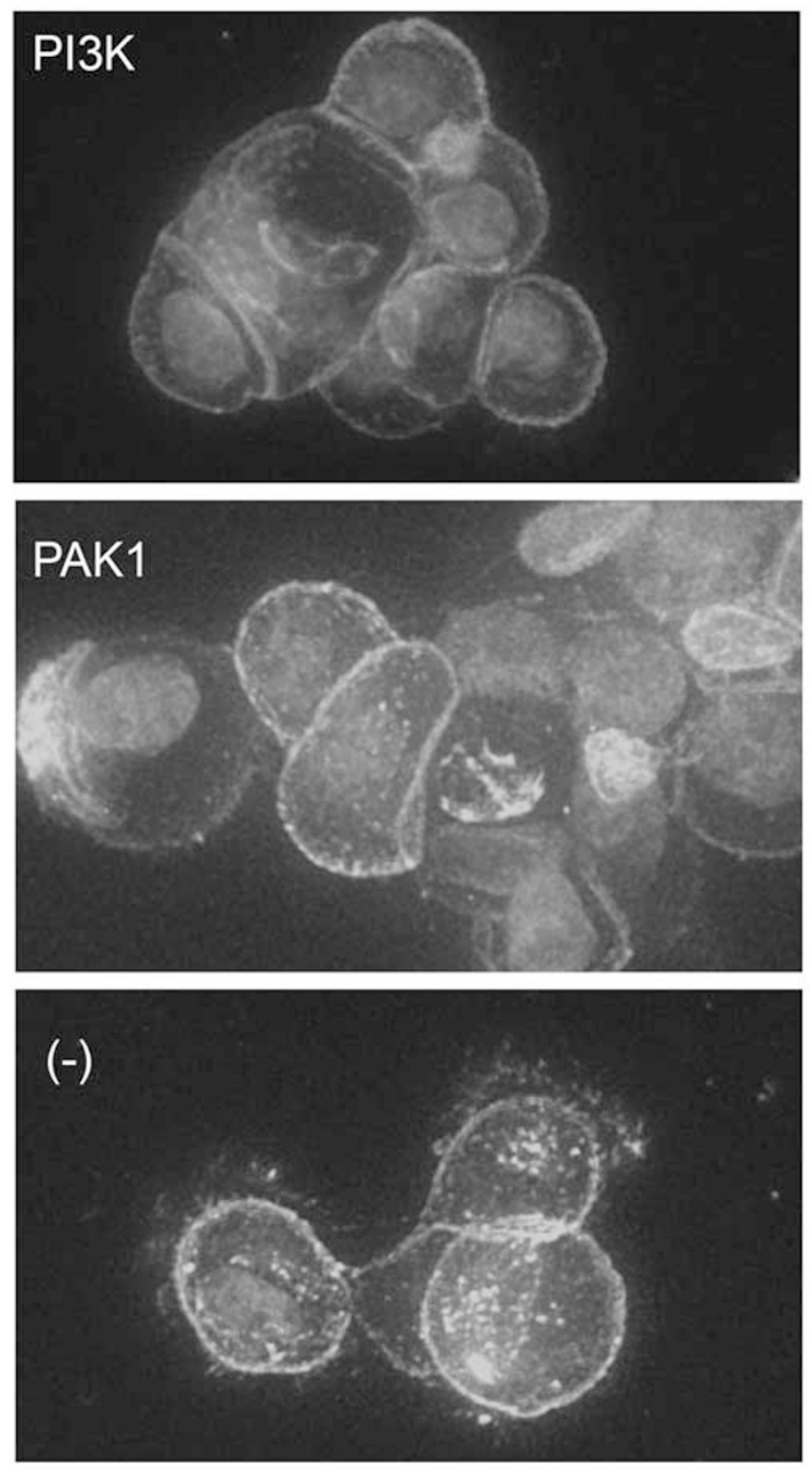
electrophoresis was performed using 6\% polyacrylamide gel containing $15-25 \mu \mathrm{M}$ Phostag (Wako Pure Chemical Industries, Osaka, Japan) according to the manufacturer's instructions. Gels were stained with Coomassie Blue or were transferred onto nitrocellulose membranes. The recombinant COL17 was generated as previously described. ${ }^{27,28}$ The recombinant COL17 was used as a reference protein, because most of the protein-makers do not work under this condition. To make dephosphorylated proteins, cell lysates were incubated with alkaline phosphatase (ALP, Sigma Aldrich, St. Louis, MO) at $37^{\circ} \mathrm{C}$ for $45 \mathrm{~min}$. Blotting was performed as described previously, ${ }^{11}$ using rabbit anti-COL17 [refs 29,30] ( $1: 2000$ dilution), rabbit anti- $\alpha 6$-integrin (Atlas Antibodies, Stockholm, Sweden, $1: 1000$ dilution), rabbit anti- $\beta 4$-integrin (Santa Cruz, Dallas, TX, 1:1000 dilution), rabbit anti- $\beta$ tubulin (Abcam, Tokyo, Japan, $1: 20000$ dilution) and mouse anti-desmoglein 3 (AbD Serotec, Kidlington, UK), as the primary antibodies, followed by HRP-conjugated goat antirabbit IgG (Life Technologies, Tokyo, Japan, 1:5000 dilution). Signals were visualized with Clarity Western ECL Substrate (Bio-Rad Laboratories, Hercules, CA).

\section{Intracellular Calcium Influx Detection}

Calcium influx was measured using Fluo 4-AM according to the manufacturer's instructions (Dojindo Molecular Technologies, Kumamoto, Japan). Briefly, DJM-1 cells were loaded with the calcium indicator Fluo $4-\mathrm{AM}$ at $37^{\circ} \mathrm{C}$ for $1 \mathrm{~h}$. After washing with PBS, cells were incubated with pre-warmed recording buffer containing probenecid. Subsequently, cells were stimulated with BP- $\operatorname{IgG}(1 \mathrm{mg} / \mathrm{ml})$ under fluorescent microscopy. Fluorescent images were obtained at an excitation wavelength of $488 \mathrm{~nm}$. The relative fluorescence intensity was measured by ImageJ (http://rsbweb.nih.gov/ij/).

\section{Statistical Analysis}

Statistical calculations were performed using SigmaPlot (Version 12.0, Systat Software, Chicago, IL). To compare the parameters, $t$-test or one-way ANOVA test was used. A $P$ value of $<0.05$ was considered statistically significant. The graphs show median \pm s.e.m.

\section{RESULTS}

Non-Hemidesmosomal COL17 is Preferentially Internalized and Depleted

We reconfirmed that the COL17 of NHEKs and DJM-1 cells were depleted by the stimulation with BP patient IgG
(BP-IgG) and mouse monoclonal anti-human COL17NC16A IgG (TS39-3) as previously reported. ${ }^{11,29,30}$ BP-IgG and TS39-3 were found to deplete COL17 in NHEKs and DJM-1 cells after $6 \mathrm{~h}$ incubation, but neither normal human IgG nor normal mouse IgG were found to cause such depletion (Figure 1a). To determine whether the internalized COL17 originated from hemidesmosomes or from nonhemidesmosomes, COL17 depletion in DJM-1 cells was separately detected in TritonX-100-soluble or TritonX-100insoluble fractions after BP-IgG stimulation. The depletion of COL17 was observed in the TritonX-100-soluble pools $6 \mathrm{~h}$ after BP-IgG treatment, whereas it was not observed in the TritonX-100-insoluble pools (Figure 1b, left). In contrast, COL17 depletion was also detected in TritonX-100-insoluble fraction $48 \mathrm{~h}$ after BP-IgG stimulation compared with normal human $\operatorname{IgG}$ (Figure $1 \mathrm{~b}$, right). These results strongly suggest that non-hemidesmosomal COL17 was preferentially depleted by stimulation with the BP-IgG in the DJM-1 cells. To confirm this, we next investigated the endocytosis and depletion of COL17 in COL17-293 cells. The expressions of transmembrane hemidesmosomal proteins were examined in COL17-293 cells. COL17 and integrin $\alpha 6$ had equivalent expressions in DJM-1 and COL17-293 cells, but integrin $\beta 4$ was hardly detected in COL17-293 cells (Figure 1c). This suggests that COL17-293 cells do not express complete hemidesmosomal proteins. Similar to DJM-1 cells, COL17 internalization induced by BP-IgG treatment was observed in COL17-293 cells (Figure 1d, lower). In addition, the amount of COL17 was reduced $24 \mathrm{~h}$ after stimulation by BP-IgG or TS39-3 (Figure 1e). Based on these results, BP-IgG can be said to induce the internalization of COL17 from TritonX-100soluble non-hemidesmosomal pools, causing a late effect of COL17 deletion from TritonX-100-insoluble hemidesmosome pools after $48 \mathrm{~h}$.

\section{Small GTPases are Involved in COL17 Macropinocytosis}

It has been shown that the endocytosis of COL17 is mediated by the macropinocytosis pathway. ${ }^{10,12}$ Macropinocytosis is generally regulated by several signaling pathways, such as those involving small GTPases, and is commonly involved in fluid uptake. It is uncertain whether the transmembrane COL17 of keratinocytes is internalized via the same signaling pathways. To address this issue, we investigated the signaling pathways. The cells were preincubated with inhibitors for $1 \mathrm{~h}$ before being stimulated with BP-IgG for $2 \mathrm{~h}$. Racl and Cdc42 inhibitors were found to strongly repress COL17 internalization, and the ROCK inhibitor also partially blocked the

Figure 2 Small GTPases are involved in macropinocytosis of COL17. The cells were preincubated with inhibitors for $1 \mathrm{~h}$, and then the cells were stimulated with BP-IgG for $2 \mathrm{~h}$. (a) IgGs stained with FTIC-conjugated anti-human IgG are detected around nuclei in the DJM-1 cells after BP-lgG stimulation (lower right). Intracellular fluorescent dots are hardly detected in the cells treated with Rac1 (upper left, trihydrochloride, $200 \mu \mathrm{M}$ ), Cdc42 (middle left, ML141, $10 \mu \mathrm{M}$ ), and ROCK (lower left, dihydrochloride, $10 \mu \mathrm{M}$ ) inhibitors. The intracellular dots are not detected in the cells treated with the inhibitor of PI3K (upper right, hydrochloride, $50 \mu \mathrm{M}$ ) which is related with small GTPase signaling, and the inhibitor of PAK1 (middle right, IPA-3, $25 \mu \mathrm{M}$ ). Scale bar, $10 \mu \mathrm{m}$. (b) Intracellular dots in the cells, at least 25 cells in each group, were quantified by ImageJ. The average dot counts is significantly lower in the cells treated with inhibitors $(P<0.05)$. 
a
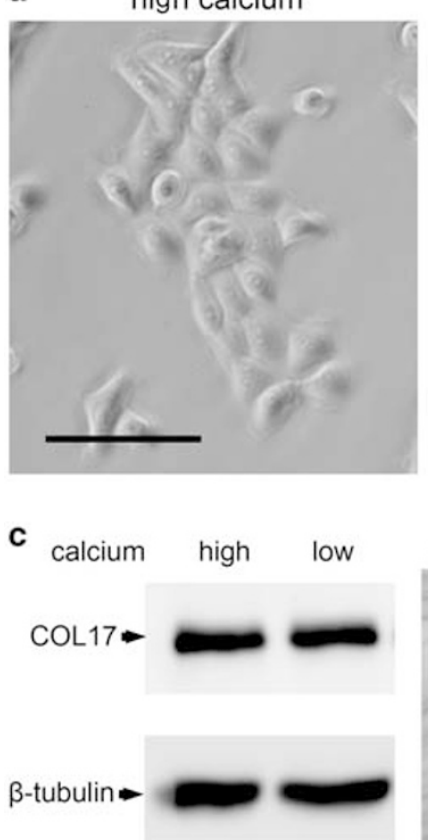

Western blot

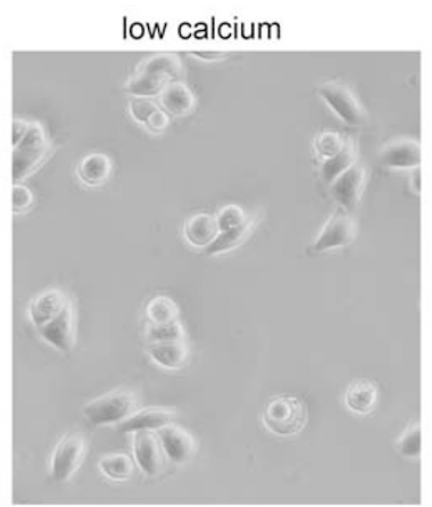

d

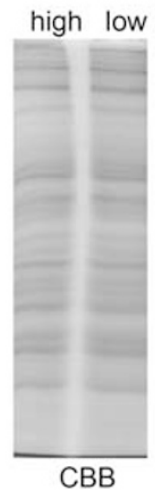

b

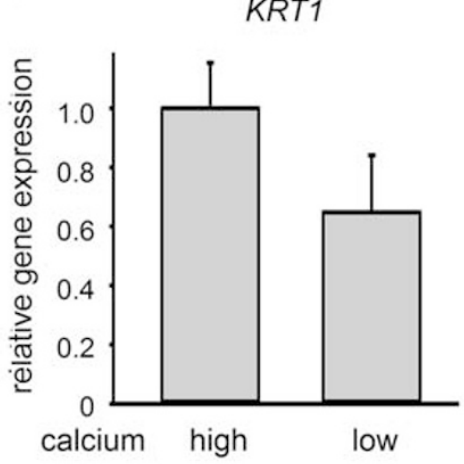

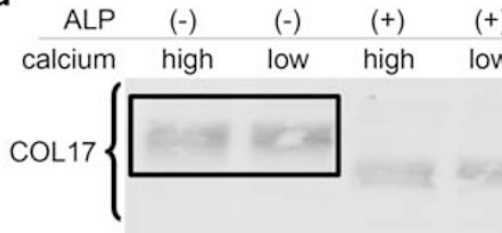

$(+)$ $(-)$

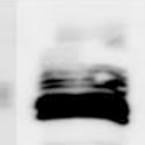

$\beta$-tubulin -
COL17HEK293

cellular purified

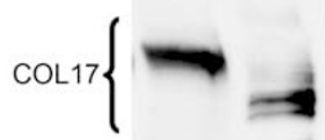

$25 \mu \mathrm{M}$ Phostag

Western blot

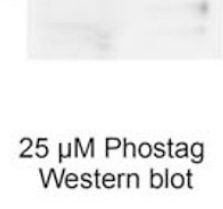

Figure 3 COL17 is phosphorylated under steady-state condition. The cells were cultured in the medium with $1.8 \mathrm{mM}$ or $0.05 \mathrm{mM}$ calcium. The morphology and colony formation of DJM-1 cells were observed. (a) Cells form colonies high-calcium (1.8 mM) medium (left), but not in low-calcium $(0.05 \mathrm{mM})$ medium (right). Scale bar, $100 \mu \mathrm{m}$. (b) The medium with high calcium (1.8 mM) induces increased KRT1 expression. (c) COL17 expression levels by western blotting (left) and the expression of other proteins by Coomassie Brilliant Blue (CBB) staining (right) do not differ between the high and low concentrations of calcium in the culture medium. (d) Western blotting using $25 \mu \mathrm{M}$ Phostag SDS-PAGE demonstrates no obvious differences in total phosphorylation levels of COL17 under high-calcium or low-calcium medium conditions. Black squares indicate COL17 phosphorylation in steadystate condition. Alkaline phosphatase (ALP) treatment results in the same levels of dephosphorylation of COL17 in both the high-calcium and lowcalcium mediums. Most of the purified recombinant COL17 protein purified from COL17-293 cells is not phosphorylated (right). (e) The purified recombinant COL17 produced by COL17-293 cells is not phosphorylated, but COL17 expressing in COL17-293 cells is phosphorylated.

endocytosis of COL17 (Figure 2a). PI3K and small GTPase are influenced each other, and COL17 internalization was found to be inhibited by PI3K inhibitor (Figure 2a). PAK1 is a downstream signal mainly activated by Cdc42 and Rac. As expected, PAK1 inhibitor blocked COL17 internalization just as the Cdc42 and Racl inhibitors did (Figure 2a). The signaling of small GTPases, such as Rac, Cdc42, and Rho, is involved in the macropinocytosis of COL17 when BP-IgG binds to COL17. Intracellular fluorescent particles were counted by ImageJ, and the number of dots was significantly lower in the cells stimulated using BP-IgG with inhibitors than in those stimulated by BP-IgG alone $(P<0.05$, Figure $2 \mathrm{~b})$.

\section{COL17 is Phosphorylated Under the Steady-State Condition}

The calcium concentration of the culture medium is known to influence the expression levels of some proteins in DJM-1 cells. ${ }^{31}$ Therefore, DJM-1 cells were cultured in DMEM with $1.8 \mathrm{mM}$ or $0.05 \mathrm{mM}$ calcium to confirm whether calcium works well in the medium. DJM-1 cells cultured in a highcalcium medium tended to form colonies, but those cultured in a low-calcium medium did not (Figure 3a). The high calcium in the medium induces KRT1 expression in keratinocytes, ${ }^{32}$ and the high calcium increased KRT1 gene expression in DJM-1 cells (Figure 3b). The concentration of calcium in the culture medium did not affect COL17 expression levels as determined by western blotting nor did it influence the expressions of other proteins as determine by Coomassie Blue staining (Figure 3c, right). Western blotting using $25 \mu \mathrm{M}$ Phostag SDS-PAGE demonstrated no obvious differences in total phosphorylation levels of COL17 under high-calcium medium $v s$ low-calcium medium (Figure 3d, left). ALP treatment resulted in the same levels of dephosphorylation of COL17 in both the high-calcium medium and the low-calcium medium (Figure 3d, middle). COL17 produced by COL17-293, the expressing COL17 in COL17293 cells were phosphorylated similarly to DJM-1 cells were phosphorylated (Figure 3e). 

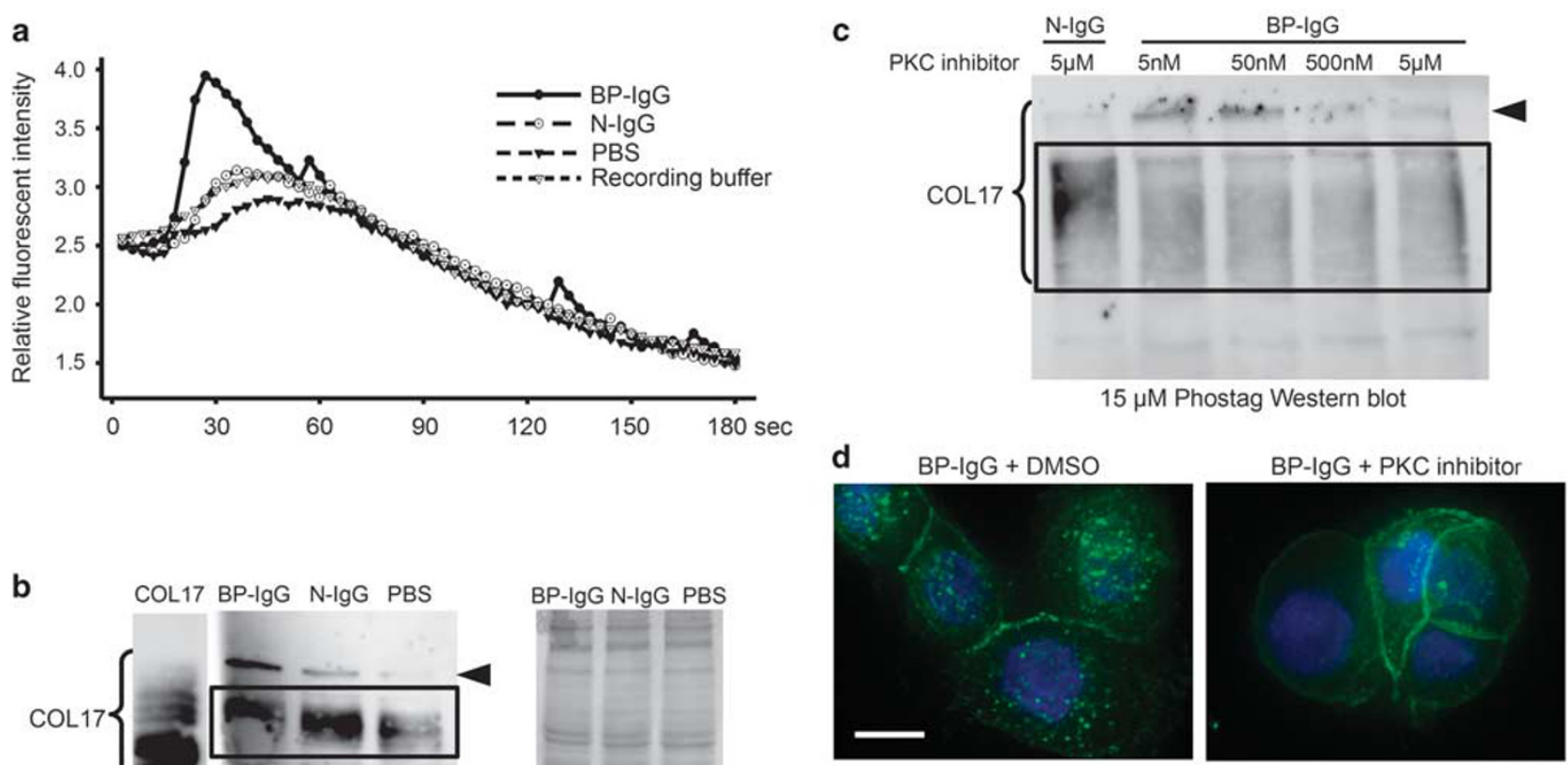

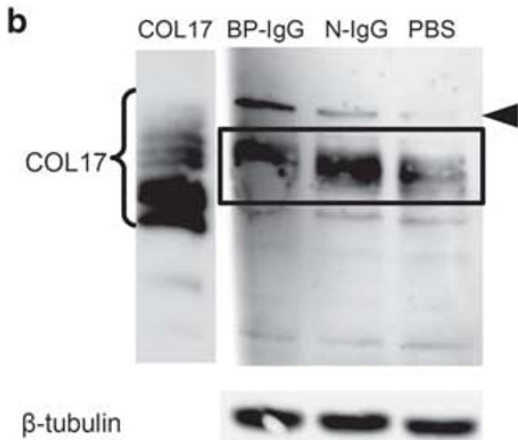

$25 \mu \mathrm{M}$ Phostag Western blot

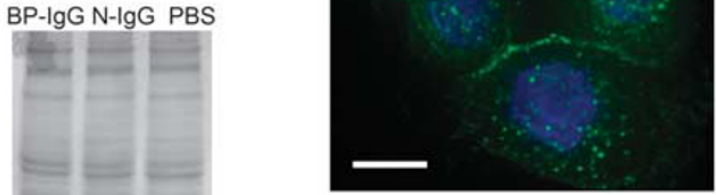

e

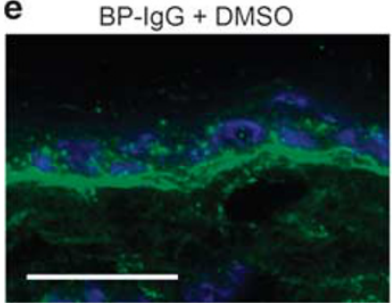

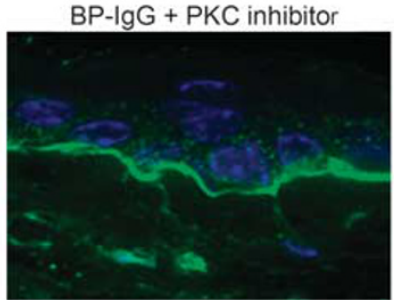

Figure 4 BP-lgG induces overabundant phosphorylation of COL17. (a) Time courses of intracellular calcium concentrations after treatments with BP-IgG, $\mathrm{N}-\mathrm{lgG}, \mathrm{PBS}$, and recording buffer as determined using fluo-4-AM. BP-lgG increases the intracellular calcium level within a minute after BP-lgG stimulation. In contrast, normal human IgG, PBS, and recording buffer do not induce a calcium influx. (b) DJM-1 cells were treated with BP-IgG, normal human IgG or PBS for $1 \mathrm{~h}$, and COL17 phosphorylation was determined by western blotting using $25 \mu \mathrm{M}$ Phostag SDS-PAGE (left). The protein expression levels are not influenced by the stimulation with IgG in Coomassie Blue staining (right). (c) DJM-1 cells were pretreated with PKC inhibitor (GF109203X), and then incubated with BP-IgG. Western blotting using $15 \mu \mathrm{M}$ Phostag SDS-PAGE shows that the PKC inhibitor protects against the overabundant phosphorylation of COL17. Black squares indicate phosphorylated-COL17 in steady-state condition, and arrows indicate overabundant phosphorylation of COL17 induced by BP-lgG in (b and $\mathbf{c}$ ). The overabundant phosphorylation of COL17 is decreased with $500 \mathrm{nM}$ and $5 \mu \mathrm{M}$ PKC inhibitor, but not with $5 \mathrm{nM}$ and $50 \mathrm{nM}$ PKC inhibitor. (d) The cells or humanized COL17 mouse ear skins was preincubated with PKC inhibitor for $1 \mathrm{~h}$, and then the cells were incubated with BP-IgG for $2 \mathrm{~h}$. IgG was detected by FITC-conjugated anti-human IgG. IgG is not detected in the cells with $5 \mu \mathrm{M}$ PKC inhibitor. (e) COL17 is detected as large dots in the cytoplasm of keratinocytes without PKC inhibitor, but not in the cells with PKC inhibitor. N-lgG, normal human IgG. Scale bar, $10 \mu \mathrm{m}(\mathbf{d})$; and $50 \mu \mathrm{m}(\mathbf{e})$.

\section{BP-IgG Induces Overabundant Phosphorylation of COL17 by PKC}

To investigate the intracellular signaling, we tested calcium influx under BP-IgG stimulation. BP-IgG increased the intracellular calcium level within a minute after BP-IgG stimulation (Figure 4a). In contrast, normal human IgG, PBS, and recording buffer did not induce calcium influx. Next, DJM-1 cells were treated with BP-IgG, normal human IgG or PBS for $1 \mathrm{~h}$, and the treatment with BP-IgG was found to induce significantly more COL17 phosphorylation than the two other treatments induced, as determined by western blotting using $25 \mu \mathrm{M}$ Phostag SDS-PAGE (Figure $4 \mathrm{~b}$, left). The protein expression levels of COL17 were not influenced in Coomassie Blue staining (Figure $4 \mathrm{~b}$, right). To clarify which kinase phosphorylates COL17 under BP-IgG stimulation, we searched for possible protein kinases by using a computer prediction system (NetPhosK 1.0 server). The in silico prediction showed that several tyrosine, serine and threonine located in intracellular domain of COL17 (Met 1 to Lys 467) might be able to be phosphorylated. In particular, certain serine (Ser 79, Ser 130, Ser367, and Ser 414) and threonine (Thr 4, Thr 412, and Thr 415) were highly expected to be phosphorylated by PKC. We and Hiroyasu et al previously reported that tyrosine kinase had no effect on the internalization of COL17 under BP-IgG treatment. ${ }^{10,12}$ Therefore, we investigated whether PKC might phosphorylate the intracellular domain of COL17 under BP-IgG stimulation. A PKC inhibitor (GF109203X) was found to protect COL17 
a

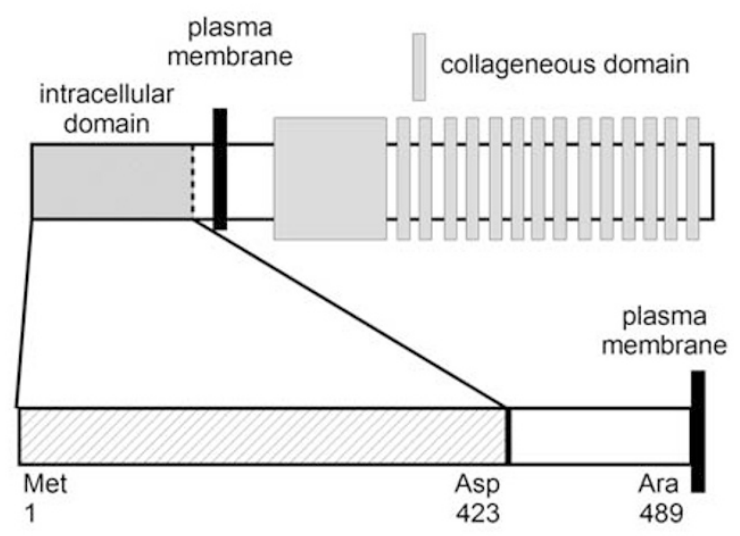

b

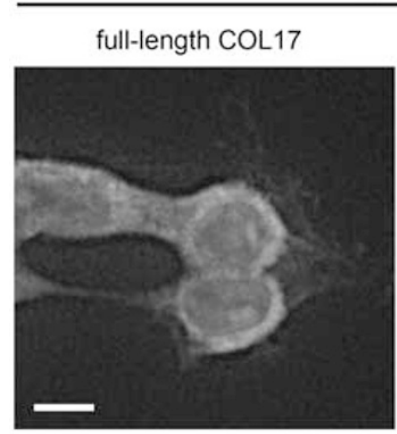

before BP-IgG stimulation

\section{COL17-delta 1-423}

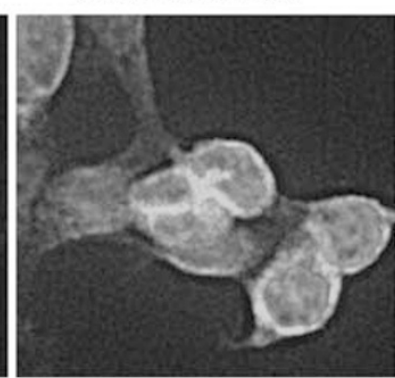

c
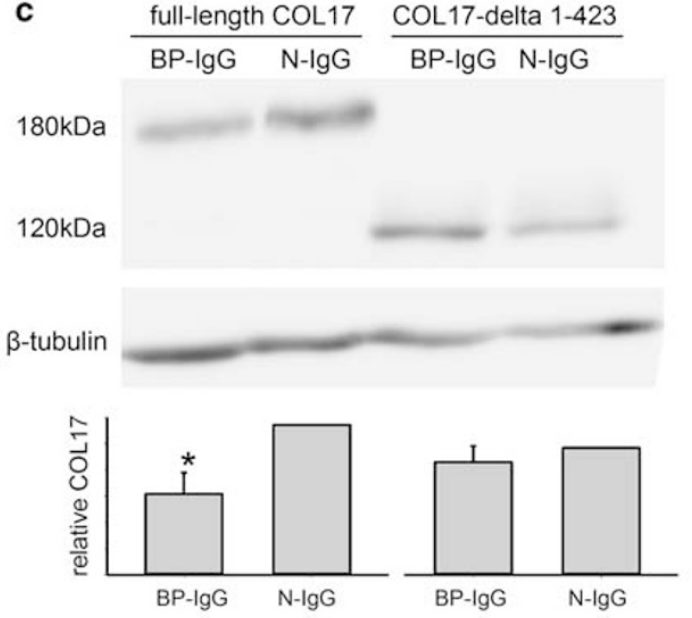

after BP-IgG stimulation

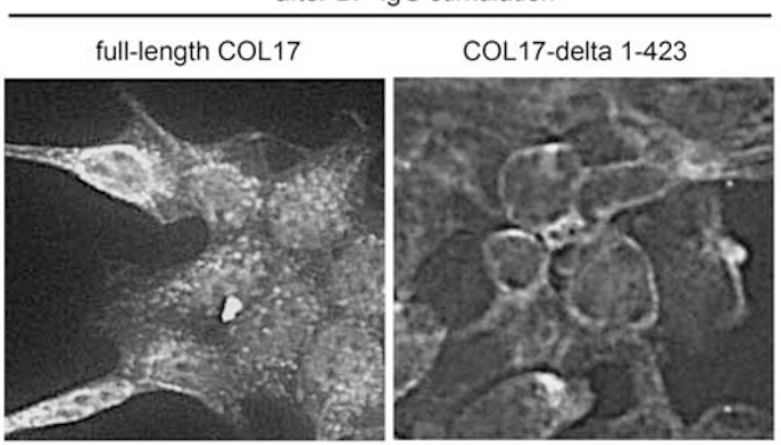

Figure 5 COL17 without intracellular domain is neither internalized nor degraded. (a) Schematic representation of molecular structure of the intracellular domain of COL17 and COL17-delta 1-423 (Met 1-Asp 423). (b) Cells were stained with BP-lgG and FITC-conjugated anti-human IgG after fixation (before BP-lgG stimulation). COL17 is detected on the plasma membrane in both COL17-293 cells and COL17-delta 1-423 cells. After BP-lgG treatment for $6 \mathrm{~h}$, cells were stained with FITC-conjugated anti-human lgG (after BP-lgG stimulation). BP-lgG is observed in the cytoplasm of full-length COL17-293 cells, but not in COL17-delta 1-423 cells. (c) Cells were treated with BP-IgG or normal human IgG for 24 h, and COL17 was detected by western blotting. COL17 is decreased in COL17-293 cells ( $\left.{ }^{*} P<0.05\right)$, but not in COL17-delta 1-423 cells. The upper panels are representative western blots, and the lower panels are relative COL17 normalized with normal IgG. Scale bar, $10 \mu \mathrm{m}$.

phosphorylation induced by BP-IgG. This overabundant phosphorylation of COL17 was decreased with $500 \mathrm{nM}$ and $5 \mu \mathrm{M}$ PKC inhibitor, but not $5 \mathrm{nM}$ and $50 \mathrm{nM}$ PKC inhibitor (Figure 4c). Furthermore, $5 \mu \mathrm{M}$ PKC inhibitor inhibited COL17 endocytosis in cell culture (Figure 4d). The blockade of COL17 endocytosis was confirmed by an organ culture system. Humanized COL17 mouse ear skin was cultured with/without PKC inhibitor, followed by incubation with BPIgG. COL17 was detected as large dots in the cytoplasm of keratinocytes, but these dots were not detected with PKC inhibitor (Figure 4e).

\section{COL17 Without Intracellular Domain are Neither Internalized Nor Degraded}

Next, we generated mutant COL17-293 cells (COL17-delta 1423 cells) which did not contain the intracellular domain (Met 1-Asp 423) (Figure 5a), and we tested whether the intracellular domain was necessary for the endocytosis of COL17. COL17-delta 1-423 cells expressed truncated COL17, which was located on the cell membrane (Figure 5b). However, the treatment of BP-IgG induced neither the endocytosis of COL17 in these cells (Figure 5b) nor the depletion of COL17 (Figure 5c).

\section{DISCUSSION}

The membranous COL17 of basal cells internalizes into cells by a macropinocytosis pathway when keratinocytes are treated with BP-IgG. ${ }^{10,12}$ It remains unclear what precise signaling in cytoskeleton reorganization is necessary for macropinocytosis after BP-IgG binding to COL17. Several membranous molecules, such as epidermal growth factor receptor (EGFR), G protein-coupled receptors (GPCRs) and integrins, are well studied in the signaling pathways, tyrosine autophosphorylation, phosphorylation of cytoplasmic domain, and activation of focal adhesion kinase, respectively. ${ }^{13,33}$ Likewise, we expected that the cytoplasmic domain of COL17 might be phosphorylated after the binding of BP-IgG. Western blotting using Phostag SDS-PAGE can 
detect the total level of phosphorylation, including phosphorylation of serine, threonine, and tyrosine. Under steadyculture conditions, COL17 of DJM-1 and COL17-transfected 293 cells were highly phosphorylated. Kitajima et al previously reported that COL17 of cultured keratinocytes was basically phosphorylated at serine residues. ${ }^{34}$ The phosphorylation was prominently increased by 12-O-tetradecanoylphorbol-13acetate treatment, which activates $\mathrm{PKC}$, and was prevented by PKC inhibitor. This indicates that COL17 is a possible substrate of PKC. Indeed, we demonstrated that PKC inhibitor prevented the overabundant phosphorylation of COL17 induced by the stimulation of BP-IgG in a dosedependent manner as determined by Phostag western blotting. Furthermore, the intracellular domain of COL17 was found to be needed for signal transduction in the macropinocytosis of COL17. These results suggest that the phosphorylation of the intracellular domain might be involved in signal transduction when COL17 internalizes into cells under BP-IgG treatment. PKC is a family of protein kinase enzymes consisting of 15 isozymes. In this study, we speculate that conventional PKC enzymes seem to be activated by BP-IgG. In general, conventional PKC requires calcium and diacylglycerol for activation, and BP-IgGinduced intracellular calcium influx occurs within a minute after stimulation.

The crucial signals for macropinosome formation were previously studied in other cells. Actin polymerization and cytoskeleton remodeling require the activation of PI3K and Rho GTPases. PI3K, Rac1, and PAK1 are particularly relevant to macropinocytosis. ${ }^{35-38}$ However, the involvement of small GTPases, such as Rac, Cdc42, and Rho, in the BP-IgGinduced macropinocytosis of COL17 remains unclear. In our experiment, small GTPase and PI3K inhibitors, including ROCK inhibitor, blocked the macropinocytosis of COL17. In contrast to previous studies, ${ }^{20,21}$ the inhibition of ROCK, a downstream signal of Rho, also partially protected the internalization of COL17 in this study. Rac1 and Cdc42 are typically involved in the formation of lamellipodia and filopodia; in contrast, Rho is mainly involved in the formation of focal adhesions and stress fibers. ${ }^{18}$ In the process of COL17 macropinocytosis, all these signaling pathways are thought to be activated after the phosphorylation of COL17 by PKC.

Hemidesmosomal COL17 consists of multiprotein complexes interacting together; therefore, it should be hard for multiprotein complexes, ie, hemidesmosomes, to enter cells by BP-IgG binding. For this assumption, we speculate that the internalization and depletion of COL17 by BP-IgG may disturb the supply of COL17 and impair the remodeling of hemidesmosomes. Eventually, these may lead to an insufficiency of functional hemidesmosomes due to a shortage of COL17. ${ }^{39}$ To test this hypothesis, Western blotting using TritonX100-soluble and -insoluble fractions was performed. In our study, the amount of COL17 in the TritonX-100soluble fraction was found to be decreased at $6 \mathrm{~h}$ after BP-IgG stimulation. In contrast, that in the TritonX-100-insoluble fraction was found to be decreased at $48 \mathrm{~h}$ after BP-IgG stimulation. These results suggest that COL17 was initially decreased in the membranous pool. A previous study demonstrated that BP-IgG treatment induces the depletion of COL17, but not the depletion of integrin $\alpha 6$ and integrin $\beta 4 .{ }^{11}$ This result suggests that hemidesmosomal multiprotein complexes do not internalize into cells under BP-IgG stimulation.

From these results and the discussion, it is tempting to speculate as follows, concerning the blistering mechanisms involved in the formation of immune complexes in BP. Initially, autoantibodies bind to COL17, which is distributed not only on hemidesmosomes but also on the plasma membrane of basal cells. Subsequently, immune complexes of COL17 and BP-IgG may be internalized by means of macropinocytosis pathways through the phosphorylation of the intracellular domain by PKC. Finally, COL17 is degraded via the ubiquitin/proteasome pathway. ${ }^{30}$ In conclusion, this depletion of COL17 from the lateral-apical plasma membrane generates a significant shortage of COL17 supplementation to hemidesmosomes, resulting in the formation of incomplete hemidesmosomes lacking COL17. Taking this into account, the specific inhibition of COL17 phosphorylation might be a potential therapeutic option.

\section{ACKNOWLEDGMENTS}

We thank Ms Mika Tanabe for her technical assistance. This work was supported in part by a JSPS Grant-in-Aid for Young Scientists (B) (26860861 to $\mathrm{HI}$ ).

\section{DISCLOSURE/CONFLICT OF INTEREST}

The authors declare no conflict of interest.

1. Schmidt E, Zillikens D. Pemphigoid diseases. Lancet 2013;381:320-332.

2. Masunaga $\mathrm{T}$, Shimizu $\mathrm{H}$, Yee $\mathrm{C}$, et al. The extracellular domain of BPAG2 localizes to anchoring filaments and its carboxyl terminus extends to the lamina densa of normal human epidermal basement membrane. J Invest Dermatol 1997;109:200-206.

3. Giudice GJ, Emery DJ, Zelickson BD, et al. Bullous pemphigoid and herpes gestationis autoantibodies recognize a common noncollagenous site on the BP180 ectodomain. J Immunol 1993;151: 5742-5750.

4. Liu Z, Giudice GJ, Zhou X, et al. A major role for neutrophils in experimental bullous pemphigoid. J Clin Invest 1997;100:1256-1263.

5. Liu Z, Giudice GJ, Swartz SJ, et al. The role of complement in experimental bullous pemphigoid. J Clin Invest 1995;95:1539-1544.

6. Hirose M, Recke A, Beckmann T, et al. Repetitive immunization breaks tolerance to type XVII collagen and leads to bullous pemphigoid in mice. J Immunol 2011;187:1176-1183.

7. Kitajima $Y$, Owaribe K, Nishizawa $Y$, et al. Phorbol ester- and calciuminduced reorganization of $180-\mathrm{kDa}$ bullous pemphigoid antigen on the ventral surface of cultured human keratinocytes as studied by immunofluorescence and immunoelectron microscopy. Exp Cell Res 1992;203:17-24.

8. Kitajima Y, Hirako Y, Owaribe K, et al. Antibody-binding to the $180-\mathrm{kD}$ bullous pemphigoid antigens at the lateral cell surface causes their internalization and inhibits their assembly at the basal cell surface in cultured keratinocytes. J Dermatol 1994;21:838-846.

9. Kitajima Y, Nojiri M, Yamada T, et al. Internalization of the $180 \mathrm{kDa}$ bullous pemphigoid antigen as immune complexes in basal keratinocytes: an important early event in blister formation in bullous pemphigoid. Br J Dermatol 1998;138:71-76. 
10. Hiroyasu S, Ozawa $\mathrm{T}$, Kobayashi $\mathrm{H}$, et al. Bullous pemphigoid IgG induces BP180 internalization via a macropinocytic pathway. Am J Pathol 2013;182:828-840.

11. Iwata H, Kamio N, Aoyama $\mathrm{Y}$, et al. IgG from patients with bullous pemphigoid depletes cultured keratinocytes of the 180-kDa bullous pemphigoid antigen (type XVII collagen) and weakens cell attachment. J Invest Dermatol 2009;129:919-926.

12. Wada M, Nishie W, Ujiie $H$, et al. Epitope-dependent pathogenicity of antibodies targeting a major bullous pemphigoid autoantigen collagen XVII/BP180. J Invest Dermatol 2016;136:938-946.

13. Sorkin A, von Zastrow M. Endocytosis and signalling: intertwining molecular networks. Nat Rev Mol Cell Biol 2009;10:609-622.

14. Kerr MC, Teasdale RD. Defining macropinocytosis. Traffic 2009;10: 364-371.

15. Haraga A, Ohlson MB, Miller SI. Salmonellae interplay with host cells. Nat Rev Microbiol 2008;6:53-66.

16. Norbury CC. Drinking a lot is good for dendritic cells. Immunology 2006;117:443-451.

17. Dubielecka PM, Cui $P$, Xiong $X$, et al. Differential regulation of macropinocytosis by Abi1/Hssh3bp1 isoforms. PLoS One 2010;5:e10430.

18. Egami $Y$, Taguchi $T$, Maekawa $M$, et al. Small GTPases and phosphoinositides in the regulatory mechanisms of macropinosome formation and maturation. Front Physiol 2014;5:374.

19. Etienne-Manneville S, Hall A. Rho GTPases in cell biology. Nature 2002;420:629-635.

20. Koivusalo $M$, Welch $C$, Hayashi $H$, et al. Amiloride inhibits macropinocytosis by lowering submembranous $\mathrm{pH}$ and preventing Rac1 and Cdc42 signaling. J Cell Biol 2010;188:547-563.

21. Patel JC, Galan JE. Differential activation and function of Rho GTPases during Salmonella-host cell interactions. J Cell Biol 2006;175:453-463.

22. Ludwig RJ, Kalies K, Köhl J, et al. Emerging treatments for pemphigoid diseases. Trends Mol Med 2013;19:501-512.

23. Li $Q$, Ujiie $H$, Shibaki $A$, et al. Human $\lg G 1$ monoclonal antibody against human collagen 17 noncollagenous $16 \mathrm{~A}$ domain induces blisters via complement activation in experimental bullous pemphigoid model. J Immunol 2010;185:7746-7755.

24. Nishie W, Sawamura D, Goto $M$, et al. Humanization of autoantigen. Nat Med 2007;13:378-383.

25. Kitajima $\mathrm{Y}$, Inoue $\mathrm{S}$, Yaoita $\mathrm{H}$. Effects of pemphigus antibody on the regeneration of cell-cell contact in keratinocyte cultures grown in low to normal Ca++ concentration. J Invest Dermatol 1987;89:167-171.
26. Nishie W, Lamer S, Schlosser A, et al. Ectodomain shedding generates neoepitopes on collagen XVII, the major autoantigen for bullous pemphigoid. J Immunol 2010;185:4938-4947.

27. Izumi K, Nishie W, Mai Y et al. Autoantibody profile differentiates between inflammatory and non-inflammatory bullous pemphigoid. J Invest Dermatol 2016;pii: S0022-202X(16)32100-5 (e-pub ahead of print 14 July 2016).

28. Nishimura $M$, Nishie $W$, Shirafuji $Y$, et al. Extracellular cleavage of collagen XVII is essential for correct cutaneous basement membrane formation. Hum Mol Genet 2016;25:328-339.

29. Natsuga K, Nishie W, Shinkuma S, et al. Antibodies to pathogenic epitopes on type XVII collagen cause skin fragility in a complementdependent and -independent manner. J Immunol 2012;188:5792-5799.

30. Ujiie H, Sasaoka T, Izumi K, et al. Bullous pemphigoid autoantibodies directly induce blister formation without complement activation. J Immunol 2014;193:4415-4428.

31. Hirako $\mathrm{Y}$, Yonemoto $\mathrm{Y}$, Yamauchi $\mathrm{T}$, et al. Isolation of a hemidesmosome-rich fraction from a human squamous cell carcinoma cell line. Exp Cell Res 2014;324:172-182.

32. Deyrieux AF, Rosas-Acosta G, Ozbun MA, et al. Sumoylation dynamics during keratinocyte differentiation. J Cell Sci 2007;120:125-136.

33. Guo W, Giancotti FG. Integrin signalling during tumour progression. Nat Rev Mol Cell Biol 2004;5:816-826.

34. Kitajima Y, Owada MK, Fujisawa $\mathrm{Y}$, et al. A hemidesmosomal transmembrane collagenous molecule, the 180-kDa bullous pemphigoid antigen (BPA II), is phosphorylated with 12-O-tetradecanoylphorbol-13-acetate in a human squamous cell carcinoma cell line (DJM-1). Epithelial Cell Biol 1995;4:70-75.

35. Lindmo K, Stenmark $H$. Regulation of membrane traffic by phosphoinositide 3-kinases. J Cell Sci 2006;119:605-614.

36. West MA, Prescott AR, Eskelinen EL, et al. Rac is required for constitutive macropinocytosis by dendritic cells but does not control its downregulation. Curr Biol 2000;10:839-848.

37. Garrett WS, Chen LM, Kroschewski R, et al. Developmental control of endocytosis in dendritic cells by Cdc42. Cell 2000;102:325-334.

38. Dharmawardhane $S$, Schürmann A, Sells MA, et al. Regulation of macropinocytosis by p21-activated kinase-1. Mol Biol Cell 2000;11: 3341-3352.

39. Iwata H, Kitajima Y. Bullous pemphigoid: role of complement and mechanisms for blister formation within the lamina lucida. Exp Dermatol 2013;22:381-385. 